\title{
A review of the relationship between eggshell colour and water vapour conductance
}

\author{
D. Charles Deeming
}

Department of Biological Sciences, University of Lincoln, Riseholme Park, Lincoln LN2 2LG, UK

E-mail: cdeeming@lincoln.ac.uk

\begin{abstract}
This review brings together the available literature that examines the effect of variations in pigmentation on the water vapour conductance of avian eggshells. Pheasant and Houbara bustard eggs provide some evidence that shell abnormalities can impact upon colour and water vapour conductance. By contrast, data from wild birds suggest that neither the degree of maculation or intensity of background colour impact upon conductance. Those studies that purport to show variation in rates of water loss in eggs with different degrees of pigmentation may have been confounded by variation in natural nesting conditions. This emerging field of research remains very much in its infancy.
\end{abstract}

Keywords: bird, eggshell, pigmentation, water vapour conductance, maculation

\section{INTRODUCTION}

Eggshell colour can be found within the body of the calcitic eggshell and/or as a superficial covering on the outer surface often associated with the shell accessory material (SAM) deposited immediately prior to oviposition (Solomon, 1987). Whilst eggshell colouration can have a range of possible functions in avian reproductive biology (see Cassey et al., 2011; Gosler et al., 2011; Sparks, 2011; Stevens, 2011; Stoddard et al., 2011), what can it tell us about the functional properties of the shell? In particular, this review will examine what we know about how colour of an eggshell reflects its ability to control gas exchange between the interior and exterior.

Eggshell porosity is typically measured as water vapour conductance $\left(\mathrm{mgH}_{2} \mathrm{O}\right.$ Torr ${ }^{-1}$ day $^{-1} ; \mathrm{Ar}$ et al., 1974) but when a group of eggs is incubated in the same environment then mass loss (typically as a percentage of initial egg mass) from each egg, which is solely due to loss of water vapour (Rahn and Paganelli, 1990), is a useful proxy measure. Whilst eggshell colour may reflect upon eggshell quality (Briggs and Williams, 1975; Campo and Escudero, 1984; Solomon, 1987) it does not reflect on shell strength, at least in domesticated species, (Solomon, 1987) although see Gosler et al. (2011) for details of a study in wild birds. There have been relatively few studies that have examined the relationship between eggshell colour and water vapour conductance. It has been suggested that the infra-red reflective properties of eggshell protoporphyrins affect eggshell temperature and affect mass loss (Gosler et al., 2005) but as far as I am aware this has never been empirically tested. However, recently as interest in the possible roles of eggshell colour in avian reproduction has increased, there have been a number of studies dealing with this critical function of the eggshell.

In this review, I bring together the details of the reports that deal with eggshell colouration and permeability, determined as water vapour conductance. Note that I do not intend to review the literature regarding the role colour may have in eggshell strength despite its correlation with eggshell thickness (Birchard and Deeming, 2009), nor am I concerned with this shell thickness directly unless it has a direct relevance to a measure for eggshell water vapour conductance.

\section{FACTORS EFFECTING CHANGES IN WATER VAPOUR CONDUCTANCE}

Eggshell porosity is typically expressed as water vapour conductance and is determined empirically by maintenance of the egg under known conditions for temperature and humidity for a period of time during which the egg mass is monitored (see Tullett, 1984). Essentially, gases diffuse through pores in the calcitic eggshell (see Figure 1 for an illustration of a pore). The rate of mass loss is essentially equivalent to loss of water vapour and so a conductance value for an eggshell can be calculated using Fick's law (Ar et al., 1974). Its value is a function of two primary physical components of the eggshell: (1) pore length, typically measured as shell thickness; and (2) the functional pore area, which is the sum of the cross- 


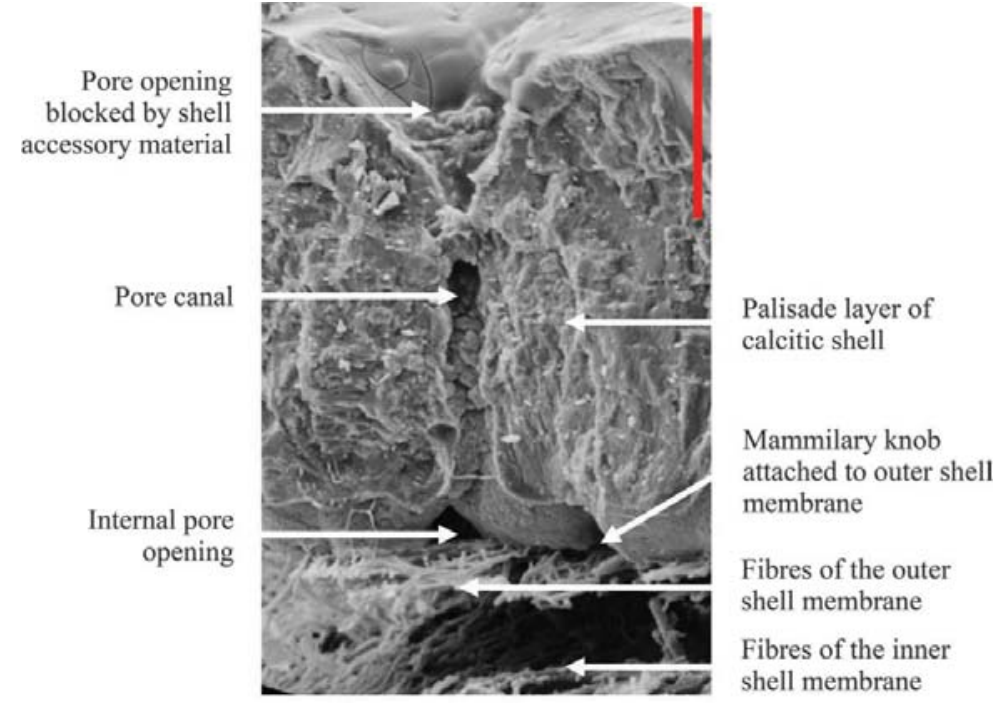

Figure 1 An example of a pore in a Houbara bustard eggshell. Scale bar $=100 \mu \mathrm{m}$.

Scanning electron micrograph by Glenn Baggott and Charles Deeming. vapour conductance almost doubles during the first seven days of incubation allowing for increased rates of water loss and exchange of oxygen (Baggott and GraemeCook, 2002).

Deeming (1987) showed that for the domestic turkey (Meleagris gallopavo) and goose (Anser anser) the artificial removal of the SAM significantly increased water vapour conductance particularly for those eggs that had initially high conductance values. By contrast, for the domestic duck (Anas platyrhynchos) removal of the SAM significantly increased conductance values at the initially low end of the range. Removal of the SAM on domestic fowl (Gallus gallus) and Muscovy duck (Cairina moschata) eggshells had no affect on water vapour conductance. The reasons for such variability was unclear but the degree of cracking within the SAM was considered to retain sectional area of each pore opening for all of the pores present in the shell (Ar and Rahn, 1985). So, a low conductance value for an eggshell may reflect a thick shell, and/or a small cross-sectional area for each pore and/or a low density of pores (number of pores per shell).

Water vapour conductance of an eggshell is generally considered as fixed at oviposition because the pores are defined by structure of the calcitic shell, which is largely unmodified during incubation. Some reports of increases in conductance during incubation are unreliable (see Deeming, 2002 for a review). In other species, such as the Malleefowl (Leipoa ocellata; Booth and Seymour, 1987) there is a real increase in conductance during incubation that is caused by etching of the inner surface of the eggshell by secretion of carbonic acid from the chorio-allantois (Simkiss, 1980). The erosion of the calcitic crystals shortens the pathway for gas diffusion (the pore length) but also importantly increases the diameter of the individual pores (Booth and Seymour, 1987).

It is known that in some species the shell accessory material (SAM) is a significant contributor to the resistance pathway that determines water vapour conductance. Effectively SAM partially blocks the pore opening and restricts gas diffusion. So, physical erosion of SAM significantly increased the water vapour conductance of eggshells in penguins (Handrich, 1989; Thompson and Goldie, 1990). In the Mandarin duck (Aix galericulata), the SAM appears to be digested away by a species of Bacillus that inhabits the external surface of the eggshell (Baggott and Graeme-Cook, 2002). This is critical to the success of natural incubation because water water vapour diffusing out of the pores and so slow down the loss of water vapour from the eggshell. In species where cracking was restricted removal of the SAM had little effect on the rate of water vapour loss.

Therefore, if eggshell pigmentation is primarily associated with the SAM then variation in the colour of eggs may reflect the extent to which the SAM is present. This is important because, if the SAM is a significant component of the resistance pathway for gas diffusion across the eggshell, then its thickness or extent of coverage may directly impact upon the water vapour conductance characteristics of the eggshell.

\section{CORRELATION BETWEEN EGGSHELL COLOUR AND WATER VAPOUR CONDUCTANCE}

\subsection{Captive birds}

Variation in eggshell colouration has been observed in the Houbara bustard (Chlamydotis undulata), (Figure 2). In this species the shell ground colour varies from a typically mid-brown overlaid with a speckling of brown patches of varying intensity, to eggshells that are almost white in colour and almost lacking any maculation (Figure 2). Baggott et al. (2003) classified eggshells into four categories: "very light", "light", "mid" and "dark" as illustrated in Figure 3. Scanning electron microscopy revealed that most of the "very light" eggshells were devoid of any SAM and the "light" eggshells had a slightly lower coverage of SAM than the other two categories. Eggshells were thicker (longer pore length) with lower which are reared for conservation purposes 


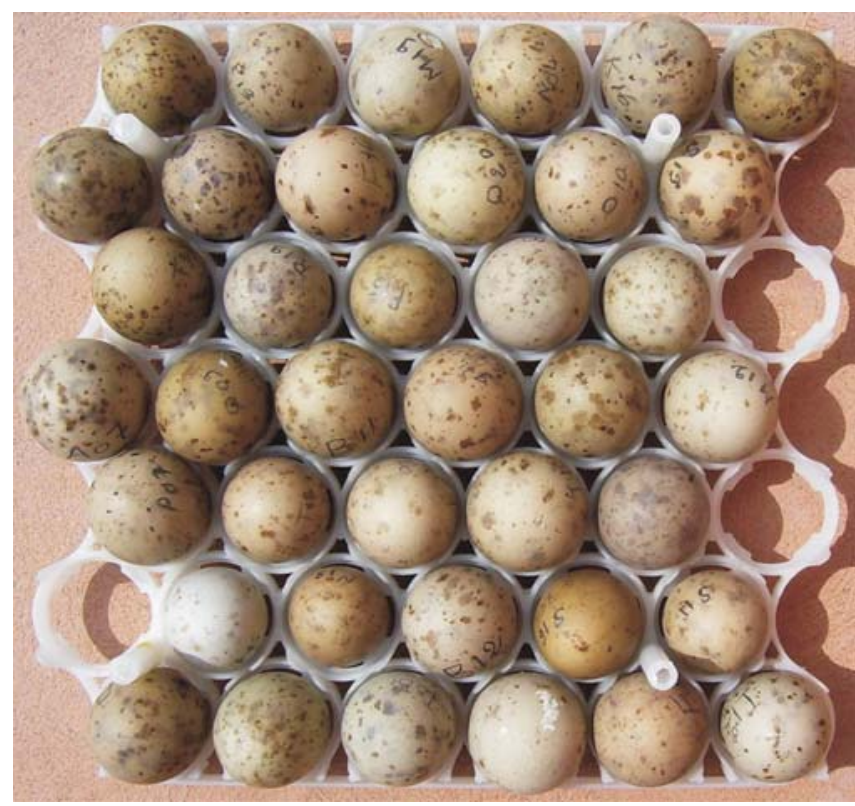

Figure 2 An example of the variation in the intensity of ground colour and maculation in Houbara bustard eggs.

Photograph by Charles Deeming.

pore density (fewer pores per eggshell) for the "mid" and "dark" categories than for the lighter shells. As a result water vapour conductance was higher in the eggshells in the "very light" and "light" categories than in the "mid" and "dark" categories. In this instance, the colour of the shell provides a useful insight into the likely porosity of an eggshell prior to incubation. This means that eggs with relatively light shells can be set in high humidity machines whereas the more normal darker eggshells would require a lower initial humidity (Baggott et al., 2003). The loss of cuticle on eggs incubated in wild nests may be crucial in causing a natural increase in gas conductance allowing for an increase in the delivery of oxygen as seen in the Mandarin duck, although in this species there is no change in eggshell colour (Baggott et al., 2003).
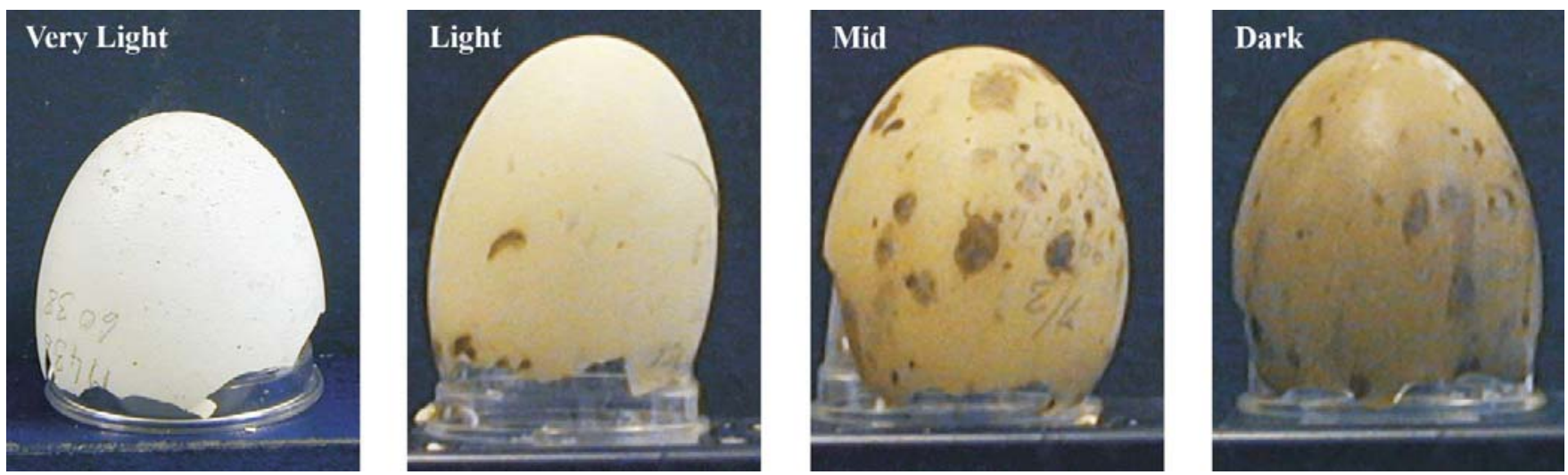

Figure 3 Examples of the categories of eggshell colouration in Houbara bustard eggs as defined by Baggott et al. (2003). Photographs courtesy of Glenn Baggott. 


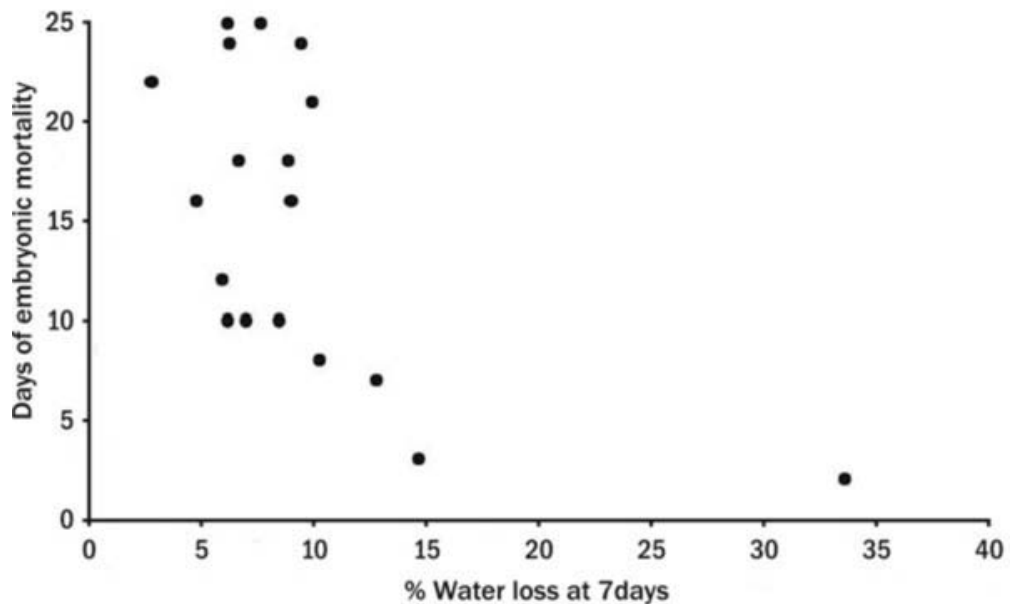

Figure 4 The relationship between rate of mass loss from ring-necked pheasant eggs with blue shells (measured as percentage reduction calculated after 7 days of incubation) and the estimated age at which embryonic mortality occurred ( $n=18$; incubation period is typically 25 days; D.C.

Deeming, unpublished data). affected by soil acidification, which resulted in limited calcium availability in the envronment (Graveland et al., 1994). The eggshells were easily recognised by their rough appearance and were significantly thinner than normal eggs. Hatchability of these deviant eggs was extremely poor (3\%) apparently caused by extreme desiccation but neither water vapour conductance nor mass loss were not recorded. This study implies that under certain conditions variability in pigmentation can indicate quality of eggshell quality.

In other populations of great tits with normal eggs whether the rate of water loss affected eggshell pigmentation depended on whether the eggs were undergoing incubation or not (Higham and Gosler, 2006). "Passive" mass loss outside of a nest and in the control artificial conditions of a refrigprocess prior to setting (Deeming, personal observation).

Studying a variety of broiler chicken breeders Joseph et al. (1999) demonstrated that the strain of bird, rather than type of feed, was more crucial in determining shell colour. Although there are significant effects of strain on rates of absolute and relative mass loss from eggs during storage, by contrast to the results seen in pheasants, there was no significant correlation for shell colour intensity and rates of mass loss for unincubated eggs from the different strains.

The red-legged partridge (Alectoris rufa) is a game bird artificially reared in large numbers in Europe for release on commercial shoots. This species exhibits variation in the degree of pigmentation ranging from light brown, probably reflecting the ground colour of the shell, through to heavily pigmented with surface speckling. Rates of water loss under the same active incubation conditions were found to be unaffected by the extent of the pigmentation: light brown, $7.4 \pm 2.4 \%$ compared with heavily pigmented, $7.6 \pm 2.5 \%$, and there was no effect of eggshell colouration on hatchability (Fraser et al., 1999). Scanning electron microscopy indicated no obvious differences in morphology of the SAM in the two groups. Colour, or degree of pigmentation, does not, therefore, necessarily reflect upon the permeability of the eggshell in all cases.

\subsection{Wild bird species}

Defective eggshells associated with deviant pigmentation have been recognised in a wild population of Great Tits (Parus major) living in a Dutch forest erator showed no significant relationship with the degree of maculation of the eggshell. By contrast, eggs that were undergoing incubation in nests exhibited a significant effect of the degree of maculation on loss of mass. This is interpreted by Higham and Gosler (2006) as the degree of maculation affecting the intrinsic property of eggshell water vapour conductance. However, there is a problem with interpretation-for instance, if the degree of maculation reflected the deposition of SAM then it could be expected that rates of water loss would correlate with the presence of SAM irrespective of the nesting environment. Eggs with a high degree of maculation would be predicted to have a lower mass loss under "passive" conditions as well as under active incubation. Similar observations have been recorded in Blue Tits (Cyanistes caeruleus) where pigmentation "spread" was significantly correlated with daily rate of mass loss during incubation (Sanz and GarcíaNavas, 2009). Despite the evidence that eggshell pores tend to have a higher density (pores per $\mathrm{mm}^{2}$ ) at the shoulder and blunt end of the egg (Rokitka and Rahn, 1987), eggs with pigment spots spread evenly across the eggshell had a lower rate of mass loss than eggs that had the maculation distribution more over the blunt pole. The presence of the spots was seen as the factor reducing rates of mass loss although there was no significant relationship between pigment intensity and rates of mass loss (Sanz and GarcíaNavas, 2009).

It would seem that Higham and Gosler (2006) and Sanz and García-Navas (2009) have not taken account of the relationship between mass loss from an egg and its value as a measure of eggshell permeability. Mass loss is a proxy for eggshell water vapour 
conductance only if all of the eggs are kept under the same humidity conditions, which cannot have been the case in the natural incubation conditions tested by Higham and Gosler (2006) or in the study by Sanz and García-Navas (2009). For instance, Great Tit nests vary in their mass and composition (Mainwaring and Hartley, 2008; Britt and Deeming, 2011), which is likely to create variation in the humidity microclimate around the eggs-a heavy, well insulated nest will retain humidity better than a less substantial, more porous nest (Deeming, 2011). If all eggs had the same water vapour conductance it is this variation that would cause differences in rates of mass loss. Between species it is known that eggshell water vapour conductance varies both within clutches and between species (Rahn and Paganelli, 1990) and can be matched to the specific nest environment (Deeming, 2011).

To determine water vapour conductance of eggs in a nest environment requires not measurement of mass loss during incubation but also a value for the partial pressure for water vapour experienced by the eggs over the same period, which can be calculated using the mass loss from an egg hygrometer of known eggshell water vapour conductance (e.g. see Rahn et al., 1977; or Swart et al., 1987). Hence, variation in mass loss of eggs incubated in real nests cannot directly represent variation in water vapour conductance of the shell because the egg will not have necessarily experienced the same humidity conditions during incubation. There is too much variation in the rates of mass loss (Rahn et al., 1977), the range of water vapour conductance of eggs between clutches (Rahn et al., 1977; Tullett, 1984; French and Tullett, 1991), or the micro-environment for temperature (Ar, 1991) and humidity within the nest caused by the nest location and construction, or the incubation behaviour of the adults causing ventilation of the nest (Rahn et al., 1977). Curiously, Higham and Gosler (2006) themselves credit some of the effects on mass loss that they observed to varying incubation behaviour of the females. Therefore, variation in rates of mass loss of eggs in multiple natural nests cannot be used as a true reflection of variation in water vapour conductance of the eggshells. Hence, like Maurer et al. (2011) I conclude that the evidence for the degree of maculation correlating with eggshell water vapour conductance is not as strong as Higham and Gosler (2006) and Sanz and García-Navas (2009) suggest.

Other studies in different species have also found no evidence for a relationship between the degree of maculation and water vapour conductance. Eggs of the black-headed gull (Chroicocephalus ridibundus) exhibit maculation on the shell as speckles of darker pigment (Maurer et al., 2011). The degree of pigmentation did not significantly affect shell thickness and water vapour conductance values for samples of shell pieces from either the speckled or plain areas did not differ significantly. Similarly, in the collared flycatcher (Ficedula albicollis) neither shell thickness nor pore density are affected by variability in shell colouration as measured in the blue-green or UV chroma (Hargitai et al., 2011).

The study by Jagannath et al. (2008) is cited (e.g. Mauer et al., 2011) as supporting a negative relationship between eggshell thickness and the degree of pigmentation (see Gosler et al., 2005, 2011). Jagannath et al. showed that in eggs from sparrowhawks (Accipiter nisus) exposed to DDT protoporphyrin spots were internalised within the palisade layer of the eggshell, which in turn was significantly thinner. This seemed to be an abnormal condition associated with the pesticide contamination but Jagannath et al. (2008) made no association with shell thickness and strength. Despite this, Maurer et al. (2011) cited this result as being equivalent to other studies regarding mass loss. However, thinner shell thickness per se does not necessarily indicate an increase in shell water vapour conductance and an increase in the rate of mass loss during incubation. Burton et al. (1987) demonstrated that in Grey Herons (Ardea cinerea) exposed to DDE the eggshells (which have no maculation) were significantly thinner, had a greater pore density and a higher water vapour conductance. However, in the nest rates of water loss from these eggs were within the normal range for heron eggs. Failure to hatch following pesticide contamination was not associated with high rates of mass loss but were caused by physical damage to abnormally thin eggshells by the adults.

\section{CONCLUSIONS}

Abnormalities in eggshell structure, for example shown in captive pheasants and Houbara bustards, can be associated with variation in the colour or degree of pigmentation of an eggshell and this can be indicative of its water vapour conductance. Hence, eggshell colouration could indicate that some eggs require a different incubation humidity environment than would be used for eggs with more typical colouration. Incubation of eggs with atypical pigmentation in higher than normal humidity may prevent early rates of desiccation that are prevalent in pheasants. However, in wild species there is no evidence to date that suggests a relationship between the degree of pigmentation on eggshells and their water vapour conductance. The studies that purport to show this (Higham and Gosler, 2006; Sanz and 
García-Navas, 2009) are flawed because rates of mass loss were incorrectly considered to be equivalent to water vapour conductance. Although it may yet be found in other species, in Great Tits at least the fact that eggshell pigmentation showed no effect of weight loss under artificial conditions (Higham and Gosler, 2006) strongly suggests that eggshell conductance does not correlate with the degree of maculation.

Eggshell pigmentation is complex because it involves a ground colour caused by pigments incorporated into the calcitic layer, and maculation that can be between calcium carbonate crystals or may be simply associated with shell accessory material. There is a need for more empirical studies that aim to distinguish between the effects on water vapour conductance associated with (a) differences in eggshell ground colour (as is the case with pheasant eggs), and (b) differences in the degree of maculation, which may be an indirect indicator of shell accessory material (as could be the case in the Houbara).

One point is clear-there is a need for a better understanding of what constitutes a change in eggshell water vapour conductance associated with a difference in shell structure. Research under field conditions needs to determine actual values for water vapour conductance of eggshells with different patterns of pigmentation rather than relying on rates of mass loss. This is an emerging field of research that could prove interesting but further careful empirical studies are needed for a range of species before we can start to understand the role played by variation in pigmentation on the gas conductance of eggshells.

\section{ACKNOWLEDGEMENTS}

I am grateful to the anonymous reviewer for constructive comments on a previous draft of this paper.

\section{REFERENCES}

Ar, A. (1991) Roles of water in avian eggs. In: Deeming, D.C. and Ferguson, M.W.J. (eds), Egg incubation: its effects on embryonic development in birds and reptiles, pp. 229-143. Cambridge University Press.

Ar, A., Paganelli, C.V., Reeves, R.B., Greene, D.G. and Rahn, H. (1974) The avian egg: water vapour conductance, shell thickness, and functional pore area. Condor, 76, 153-158.

Ar, A. and Rahn, H. (1985). Pore in avian eggshells: gas conductance, gas exchange and embryonic growth rate. Resp. Physiol., 61, 1-20.
Baggott, G.K., Deeming, D.C., Hémon, S. and Paillat, P. (2003). Relationships between eggshell pigmentation, ultrastructure and water vapour conductance in the Houbara bustard (Chlamydotis undulata macqueeni). In: Scullion, F.T. and Bailey, T.A. (eds), Proc. World Ass. Wildl. Vet. Wildlife Sess. 27th World Vet. Congr., Tunisia, 26th September 2002, pp. 85-88. World Association of Wildlife Veterinarians, Northern Ireland.

Baggott, G.K. and Graeme-Cook, K. (2002) Microbiology of natural incubation. In: Deeming, D.C. (ed.), Avian incubation: behaviour, environment and evolution, pp. 179-191. Oxford University Press.

Birchard, G.F. and Deeming, D.C. (2009) Scaling of avian eggshell thickness: implications for maximum body mass in birds. J. Zool., 279, 95-101.

Booth, D.T. and Seymour, R.S. (1987) Effect of eggshell thinning on water vapor conductance of malleefowl eggs. Condor, 89, 453-459.

Briggs, D.M. and Williams. C.M. (1975) Shell strength, hatchability, egg production, egg production and egg shell pigmentation in the Japanese quail. Poult. Sci., 54, 1738.

Britt, J. and Deeming, D.C. (2011) First egg date and air temperature affect nest construction in Blue Tits Cyanistes caeruleus but not in Great Tits Parus major. Bird Study, 58, $78-89$.

Burton, F.G., Marquiss, M. and Tullett, S.G. (1986) A note on eggshell porosity, nest humidity and the effects of DDE in the grey heron (Ardea cinerea). Comp. Biochem. Physiol., C85, $25-31$.

Campo, J.L. and Escudero, J. (1984) Relationship between egg shell colour and two measurements of shell strength in the Vasca breed. Brit. Poult. Sci., 25, 467-476.

Cassey, P., Maurer, G., Lovell, P.G. and Hanley, D. (2011) Conspicuous eggs and colourful hypotheses: testing the role of multiple influences on avian eggshell appearance. Avian Biol. Res., 4, 185-195.

Deeming, D.C. (1987) Effect of cuticle removal on the water vapour conductance of egg shells of several species of domestic bird. Brit. Poult. Sci., 28, 231-237.

Deeming, D.C. (2002) Functional characteristics of eggs. In: Deeming, D.C. (ed.), Avian incubation: behaviour, environment and evolution, pp. 28-42. Oxford University Press.

Deeming, D.C. (2011) Importance of nest type on the regulation of nest humidity in birds. Avian Biol. Res., 4, 23-31.

Deeming, D.C., Hodges H.R. and Cooper, J.J. (2011) Effect of sight barriers in pens of breeding ring-necked pheasants (Phasianus colchicus): II. Reproductive parameters. Brit. Poult. Sci., 52, 415-422.

Deeming, D.C. and Wadland, D. (2002) Influence of mating sex ratio in commercial pheasant flocks on bird health and the production, fertility, and hatchability of eggs. Brit. Poult. Sci., 43, 16-23.

Fraser, A.C., Cusack, M. and Deeming, D.C. (1999). Ultrastructure of red-legged partridge (Alectoris rufa) eggshells. Int. Hatch. Pract., 14(2), 19.

French, N.A. and Tullett, S.G. (1991) Variation in the eggs of poultry species. In: Tullett, S.G. (ed.), Avian Incubation, pp. 59-77. Butterworths-Heinemann, London.

Gosler, AG., Higham, J.P. and Reynolds, S.J. (2005) Why are birds' eggs speckled? Ecol. Lett., 8, 1105-1113. 
Gosler, A., Connor, O. and Bonsar, R. et al. (2011) Protoporphyrin pigment contributes to eggshell strength in a passerine bird. Avian Biol. Res., 4, XXXX.

Graveland, J., van der Wal, R., van Balen, J.H. and van Noordwijk, A.J. (1994) Poor reproduction in forest passerines from decline of snail abundance on acidified soils. Nature, 368, 446-448.

Handrich, Y. (1989). Incubation water loss in King penguin egg. I. Change in egg and brood pouch parameters. Physiol. Zool., 62, 96-118.

Hargitai, R., Mateo, R. and Török, J. (2011) Shell thickness and pore density in relation to shell colouration, female characteristics and environmental factors in the collared flycatcher (Ficedula albicollis). J. Ornithol., 152, 579-588.

Higham, J.P. and Gosler, A.G. (2006) Speckled eggs: water-loss and incubation behaviour in the great tit Parus major. Oecologia, 149, 561-570.

Hulet, R.H., Flegal, C.J., Carpenter, G.H. and Champion, L.R. (1985) Effect of eggshell color and thickness on hatchability in Chinese ring-necked pheasants. Poult. Sci., 64, 235-237.

Joseph, N.S., Robinson, N.A., Renema, R.A. and Robinson, F.E. (1999) Shell quality and color variation in broiler breeder eggs. J. Appl. Poult. Res., 8, 70-74.

Kożuszek, R., Kontecka, H., Nowaczewski, S. and Rosiński, A. (2009). Storage time and eggshell colour of pheasant eggs vs. the number of blastodermal cells and hatchability results. Folio Biolog. (Kraków), 57, 121-130.

Mainwaring, M.C. and Hartley, I.R. (2008) Seasonal adjustments in nest cup lining in Blue tits Cyanistes caeruleus. Ardea, 96, 278-282.

Maurer, G., Portugal, S.J., Mikšik, I and Cassey, P. (2011). Speckles of cryptic black-headed gull eggs show no mechanical or conductance structural function. J. Zool., doi: 10.1111/j.1469-7998.2011.00830.x.

Rahn, H., Ackerman, R.A. and Paganelli, C.V. (1977) Humidity in the avian nest and egg water loss during incubation. Physiol. Zool., 50, 269-283.

Rahn, H. and Paganelli, C.V. (1990) Gas fluxes in avian eggs: driving forces and the pathway for exchange. Comp. Biochem. Physiol., 95A, 1-15.
Richards, P.D.G. and Deeming, D.C. (2000a). Correlation between shell colour and ultrastructure in pheasant eggs. Avian Poult. Biol. Rev., 11, 305-306.

Richards, P.D.G. and Deeming, D.C. (2000b) The mammillary layer-OSM interface in blue pheasant eggshells. Micro. Soc. S. Afr., Proc., 30, 64.

Richards, P.D.G. and Deeming, D.C. (2001) Correlation between shell colour and ultrastructure in pheasant eggs. Brit. Poult. Sci., 42, 338-343.

Rokitka, M.A. and Rahn, H. (1987) Regional differences in shell conductance and pore density of avian eggs. Resp. Physiol., 68, $371-376$.

Sanz, J.J. and García-Navas, V. (2009) Eggshell pigmentation pattern in relation to breeding performance of blue tits Cyanistes caeruleus. J. Anim. Ecol., 78, 31-41.

Simkiss, K. (1980) Water and ion fluxes inside the egg. Am. Zool., 20, 385-393.

Solomon, S.E. (1987) Egg shell pigmentation. In: Wells, R.G. and Belyavin, C.G. (eds), Egg quality: current problems and recent advances, pp. 147-157. Butterworths, London.

Sparks, N.H.C. (2011) The chemical nature and production of egg colouration. Avian Biol. Res., 4, 162-167.

Stevens, M. (2011) Avian vision and egg colouration: concepts and measurements. Avian Biol. Res., 4, 168-184.

Stoddard, M.C., Marshall, K. and Kilner, R. (2011) Imperfectly camouflaged avian eggs: artefact or adaptation? Avian Biol. Res., 4, 196-213.

Swart, D., Rahn, H. and de Kock, J. (1987) Nest microclimate and incubation water loss of eggs of the African ostrich (Struthio camelus var. domesticus). J. Exp. Zool., Suppl. 1, $239-246$.

Thompson, M.B. and Goldie, K.N. (1990) Conductance and structure of eggs of Adélie penguins, Pygoscelis adeliae, and its implications for incubation. Condor, 92, 304-312.

Tullett, S.G. (1984) The porosity of avian eggshells. Comp. Biochem. Physiol., 78A, 5-13. 